\title{
The relationship between clinical characteristics and psychological status and quality of life in patients with vasovagal syncope
}

\author{
(1) Adem Atici, ${ }^{1}$ (1) Ramazan Asoglu, ${ }^{2}$ (i) Ahmet Demirkiran, ${ }^{3}$ (i) Nail Guven Serbest, ${ }^{3}$ (i) Baris Emektas, ${ }^{3}$ \\ (D) Remzi Sarikaya, ${ }^{3}$ (D) Ipek Yeldan, ${ }^{4}$ (D) Ahmet Kaya Bilge ${ }^{3}$ \\ ${ }^{1}$ Department of Cardiology, Istanbul Medeniyet University, Istanbul, Turkey \\ ${ }^{2}$ Department of Cardiology, Adiyaman University Training and Research Hospital, Adiyaman, Turkey \\ ${ }^{3}$ Department of Cardiology, Istanbul Medical Faculty, Istanbul, Turkey \\ ${ }^{4}$ Department of Physical Therapy, Bakirkoy Sadi Konuk Hospital, Istanbul, Turkey
}

\begin{abstract}
OBJECTIVE: Syncope is characterized by sudden and short-term loss of postural tone and consciousness. Vasovagal syncope (VVS) episode is usually self-limiting, and it may affect patients' quality of life. In this study, we aimed to investigate the relationship between quality of life, Beck-depression and Beck-anxiety scales and clinical characteristics of the VVS patients.

METHODS: The present study included 88 patients with VVS. Tilt table test was performed to all the patients. Patients filled out the Quality of life, Beck-depression, and Beck-anxiety scale forms. Demographics, medical history, echocardiography, blood pressures, electrocardiography and physical examination findings were recorded.

RESULTS: There was a significant and negative correlation between the total syncope episodes (TSE) and EQ-5D index and EQ-5D-VAS index, respectively $(p<0.001, r:-0.649 ; p<0.001, r:-0.587)$. TSE was significantly and positively correlated with the Beck-anxiety scale and Beck-depression scale. EQ-5D index, EQ-5D-VAS index, Beck-depression scale, and Beck-anxiety scales were defined as an independent predictor of TSE in VVS patients.

CONCLUSION: Psychological factors play an essential role in VVS patients. EQ-5D index, EQ-5D-VAS index, Beck-depression, and Beck-anxiety scales were an independent predictor of the TSE in patients with VVS.

Keywords: Psychological status; quality of life; vasovagal syncope.

Cite this article as: Atici A, Asoglu R, Demirkiran A, Serbest NG, Emektas B, Sarikaya R, et al. The relationship between clinical characteristics and psychological status and quality of life in patients with vasovagal syncope. North Clin Istanb 2020;7(3):237-245.
\end{abstract}

Cyncope is characterized by sudden and short-term Oloss of postural tone and consciousness [1]. Approximately $30 \%$ of the population has a syncope episode during their lifetime [2], and the most common cause is vasovagal syncope (VVS) [3]. VVS episode is usually self-limiting, but it may affect patients' quality of life and leads to disability due to falls [4-6]. Although the patho- physiological mechanisms underlying VVS are not fully understood, it may be explained by the lack of adaptive mechanisms that the regulation of the systemic blood pressure and the blood supply to the brain [7]. Tilt table test is generally used for the diagnosis of VVS [8].

Stimulation of the limbic system, which is one of the main adaptive mechanisms, maybe causing VVS in case

Received: July 30, 2019 Accepted: January 14, 2020 Online: February 10, 2020

Correspondence: Dr. Adem ATICI. Istanbul Medeniyet Universitesi, Goztepe Egitim ve Arastirma Hastanesi, Kardiyoloji Klinigi, Istanbul, Turkey.

Tel: +90 5549882969 e-mail: adematici10@gmail.com

(c) Copyright 2020 by Istanbul Provincial Directorate of Health - Available online at www.northclinist.com 
of emotional stress and may explain the pathophysiology related to VVS [9]. In the case of emotional stress related to VVS, some cortical areas show the reaction to the primary triggers, such as sorrow and pain. The endogenous opioid and serotonin play an essential role in the vasodepressor response in these cortical areas $[10,11]$. Serotonin molecule reduces sympathetic activity and increases parasympathetic activity with central effects, and serotonin reuptake inhibitors (SRRI) are considered an effective treatment in VVS patients [12].

Previous studies investigated the effects of psychiatric problems, such as anxiety on VVS. Especially, anxiety and depression were the most common mental disorders in these study groups [13-16]. The anxiety and depression that arise from VVS or VVS was a result of these mental disorders [17]. To understand the psychological status clearly and its contribution to VVS treatment, in this study, we aimed to investigate the relationship between quality of life, Beck-depression and Beck-anxiety scales and clinical characteristics of VVS patients.

\section{MATERIALS AND METHODS}

\section{Study Population}

Between May 2016 and June 2017, 88 patients with VVS in cardiac arrhythmia clinic were included in this study. Patients were higher than 18 years of age and had at least three syncope attacks. Exclusion criteria were as follows: having structural heart disease, sick sinus syndrome, pseudo-syncope, carotid hypersensitivity, intraventricular conduction disorder, orthostatic hypotension, atrial fibrillation, metabolic disorder, neurological disorders and history of antiarrhythmic medication usage for any reason. Syncope was defined as a transient loss of consciousness with a brief period of unresponsiveness and a loss of postural tone, resulting in spontaneous recovery without any resuscitation. Presyncope is defined as extreme dizziness, visual sensations, such as the disappearance of peripheral view with "tunnel view" or "blackouts" and various conscious disorders without loss of consciousness. Pseudo-syncope is defined as a disorder that may occur without prominent cardiac, reflex, neurological, or metabolic causes, and it is not an exact loss of conscious disorder [18]. A pseudo-syncope diagnosis is made when a patient shows altered voluntary motor and/or sensory symptoms that are not consistent with known neurological or medical pathology.
Quality of life, Beck's depression and anxiety scale forms were filled out by the patients in a quiet room and compared the relationship between patients' clinical characteristics. Demographics, medical history, echocardiography, blood pressures, electrocardiography and physical examination findings were recorded. Investigations included surface electrocardiography, $24 \mathrm{~h}$ blood pressure and $48 \mathrm{~h}$ rhythm Holter monitoring, exercise stress testing, and routine neurological assessments; all of these were normal. The local ethics committee approved this study, and all patients gave informed consent (date: 18.01.2016; number: 20302).

\section{Head-up Tilt Test Protocol}

The head-up tilt test was always performed in the morning (after overnight fasting) between 9:00 A.M. and 12:OO noon to avoid any possible influence of diurnal variation on autonomic tone. The study room was quiet with dimmed lights. Informed consent was obtained from all the patients. The tilt table used was an electrically motorized bed with a footboard support and 15 seconds of travel time between 0 and $60^{\circ}$. The specific head-up tilt test protocol consisted of frequent (every five minutes or more often) blood pressure and heart rate measurements during an initial period of supine rest for 10 minutes and during a subsequent period of head-up tilt to $60^{\circ}$ for 45 minutes or until symptoms developed. As soon as syncope or pre-syncope occurred, the patient was rapidly returned to the supine position with immediate restoration of consciousness and without any adverse equal. During the test, the blood pressure was measured by cuff sphygmomanometer and the heart rate was measured by continuous electrocardiographic recording [19]. Also, isoproterenol was infused at a rate of $0.05 \mu \mathrm{g} /$ $\mathrm{kg} / \mathrm{min}$ (not exceeding the maximal dose of $5 \mu \mathrm{g} / \mathrm{min}$ ) for five minutes, if necessary. Syncope of patients with a positive Head-up Tilt Test (HUT) test was classified based on the modified Vasovagal Syncope International Study (VASIS) classification as VASIS 1 (mixed), VASIS 2A (cardioinhibition without asystole), VASIS 2B (cardioinhibition with asystole) and VASIS 3 (vasodepressive) [20].

\section{EQ-5D Quality of Life Scale}

The EQ-5D is a quality of life measurement scale, which was developed by the EuroQoL group (the Western European quality of life research community) and assesses five dimensions with five questions. The five dimensions are mobility, self-care, usual activities, pain/ discomfort and anxiety/depression. Each dimension has 
three levels, including no problems, some problems and extreme problems. With this scale, 243 different health states and between -0.59 to 1 index scores could obtain. In index scores, a value of 1 indicates perfect health and 0 indicates death, and negative values indicate some clinical situations, such as being unconscious or dependent on the bed. There is a visual analog scale (VAS) (EQVAS) in the EQ-5D scale that includes answers related to the health condition. In the EQ-VAS scale, there is a measurement from 0 to 100 that a value of 0 indicates "the worst state of health imaginable" and a value of 100 indicates "the best health condition imaginable" [21].

\section{Beck-Depression Scale}

Dr. Aaron T. Beck created the Beck-depression scale, in which the questionnaire contains 21 multiple choice questions to determine the severity of depression. Emotional symptoms (e.g., despair and guilt) and physical symptoms (e.g., fatigue and weight loss) were evaluated in this questionnaire. The past failures and mistakes, pessimism, feelings of guilt, punishment and worthlessness, self-esteem, self-criticism, and suicidal thoughts were evaluated in the emotional sub-scale. Also, in the somatic sub-scale, the sadness, loss of pleasure, crying, agitation, apathy, indecision, loss of energy, changes in sleep patterns, irritability, change in appetite, difficulty in concentration, fatigue, and loss of sexual desire were evaluated. The lowest score was 0 , and the highest score was 3 for each question in the sub-scale questionnaire. Total scores from 0 to 9 points indicate no or minimal depressive symptoms, 10 to 16 points indicate mild depressive symptoms, 17 to 29 points indicate moderate depressive symptoms, and 30 to 63 points indicate severe depressive symptoms, as described previously [22].

\section{Beck-Anxiety Scale}

The Beck-anxiety scale is an internationally used anxiety scale, in which the questionnaire contains 21 multiple choice questions to determine the severity of anxiety. The beck-anxiety scale assesses the anxiety symptoms (such as numbness or tingling, sweating not due to heat, fear of the losing control, irritability, indigestion, or discomfort in the stomach or choking sensation). The patients answered the questions with one of the four choices (no, mild, moderate, severe). In addition, each answer was evaluated the severity of anxiety with points, such as 0 point indicates no, 1 point indicates mild, 2 points indicate middle, and 3 points indicates severe. All points
TABLE 1. Demographic and clinical characteristics of the 88 patients with VVS

\begin{tabular}{|c|c|}
\hline Age (years) & $34.7 \pm 14.0$ \\
\hline BMI $\left(\mathrm{kg} / \mathrm{m}^{2}\right)$ & $23.5 \pm 4.1$ \\
\hline Women $(\%)$ & 61 \\
\hline Ejection fraction (\%) & $65.2 \pm 7.7$ \\
\hline Heart rate (bpm) & $77.6 \pm 9.7$ \\
\hline $\mathrm{SBP}(\mathrm{mm} \mathrm{Hg})$ & $110.9 \pm 12.8$ \\
\hline $\mathrm{DBP}(\mathrm{mm} \mathrm{Hg})$ & $71.8 \pm 8.3$ \\
\hline TSS & $10.1 \pm 4.8$ \\
\hline Test duration (minutes) & $29.6 \pm 13.0$ \\
\hline Duration of asystole (second) & 11 \\
\hline \multicolumn{2}{|l|}{ VV Type } \\
\hline Type-1 (\%) & 49 \\
\hline Type-2 (\%) & 17 \\
\hline Type-3 (\%) & 34 \\
\hline \multicolumn{2}{|l|}{ Scales } \\
\hline EQ-5D index & $0.48 \pm 0.22$ \\
\hline EQ-5D vas & $73.6 \pm 14.3$ \\
\hline Beck-Depression & $19.4 \pm 8.7$ \\
\hline Beck-Anxiety & $16.3 \pm 7.0$ \\
\hline Minimal-depression (\%) & 3 \\
\hline Mild-depression (\%) & 7 \\
\hline Moderate-depression (\%) & 78 \\
\hline Severe-depression n (\%) & 12 \\
\hline Minimal-anxiety (\%) & 6 \\
\hline Mild-anxiety (\%) & 12 \\
\hline Moderate-anxiety (\%) & 65 \\
\hline Severe-anxiety (\%) & 17 \\
\hline
\end{tabular}

BMI: Body mass index; SBP: Systolic blood pressure; DBP: Diastolic blood pressure; TSS: Total syncope number; VVS: Vasovagal syncope.

collected after completion of the questionnaire. Scale indicating the severity of anxiety in the following way: minimal ( $0-7$ points), mild ( $8-15$ points), moderate (16-25 points) and severe (26-63 points) [22].

\section{Statistical Analysis}

Data were analyzed using SPSS software version 20.0 for Windows (SPSS Inc, Chicago, Illinois). The Kolmogorov-Smirnov test and Shapiro Wilk tests were used to verify that continuous variables were normally distributed. Normally distributed variables were expressed as mean \pm standard deviation (SD), while non-normally distributed variables were expressed as median with interquartile range (IQR). The categorical variables were presented as percentages. The relationship between vaso- 
TABLE2. Demographic and clinical characteristics of the vasovagal syncope patients' types

\begin{tabular}{lcccc} 
& Type-I $(\mathrm{n}=43)$ & Type-II $(\mathrm{n}=30)$ & Type-III $(\mathrm{n}=15)$ & $\mathrm{p}$ \\
\hline Age (years) & $36.7 \pm 14.2$ & $35.4 \pm 14.4$ & $27.5 \pm 10.9$ & 0.08 \\
Body mass index $\left(\mathrm{kg} / \mathrm{m}^{2}\right)$ & $23.5 \pm 4.1$ & $23.1 \pm 4.1$ & $24.3 \pm 4.5$ & 0.65 \\
Female (\%) & 65 & 56 & 60 & 0.76 \\
Ejection fraction (\%) & $64.1 \pm 5.5$ & $65.2 \pm 9.5$ & $65.9 \pm 7.5$ & 0.74 \\
Heart rate (bpm) & $78.1 \pm 11.7$ & $78.0 \pm 8.1$ & $75.6 \pm 6.5$ & 0.68 \\
SBP (mm Hg) & $111.9 \pm 12.5$ & $108.5 \pm 13.0$ & $113.3 \pm 13.5$ & 0.41 \\
DBP (mm Hg) & $71.2 \pm 9.0$ & $71.8 \pm 8.6$ & $73.3 \pm 6.2$ & 0.71 \\
TSS & $10.5 \pm 4.8$ & $9.8 \pm 5.3$ & $9.5 \pm 4.6$ & 0.76 \\
Test duration (minutes) & $32.9 \pm 9.7$ & $29.5 \pm 13.0$ & $28.1 \pm 10.5$ & 0.11 \\
EQ-5D index & $0.3 \pm 0.3$ & $0.4 \pm 0.3$ & $0.3 \pm 0.3$ & 0.47 \\
EQ-5D vas & $17.8 \pm 7.1$ & $73.3 \pm 12.3$ & $77.7 \pm 11.2$ & 0.48 \\
Beck-depression & $72.4 \pm 16.6$ & $18.5 \pm 10.1$ & $20.3 \pm 7.8$ & 0.75 \\
Beck-anxiety & $19.8 \pm 8.2$ & $14.8 \pm 7.6$ & $15.4 \pm 4.7$ & 0.15 \\
\hline SBP: Systic bor. & & & \\
\hline
\end{tabular}

SBP: Systolic blood pressure; DBP: Diastolic blood pressure; TSS: Total syncope number.

vagal syncope types and general quality of life, the Beck-depression and Beck-anxiety scale was evaluated using ANOVA or Kruskal Wallis test. As the dependent variables were constant, multiple linear regression analysis was used to assess the correlation between dependent and independent variables. The correlation between TSE and general quality of life, the beck-depression and beck-anxiety scale was examined using Pearson's correlation (twotailed). Statistical significance was defined as $\mathrm{p}<0.05$.

\section{RESULTS}

Table 1 shows the demographic and clinical characteristics of the 88 patients. The patients' mean age was $34.70 \pm 14.02$ years, body mass index was $23.50 \pm 4.12 \mathrm{~kg} / \mathrm{m}^{2}$ and 54 of them were female. The ejection fraction was in the normal range of $65.21 \pm 7.72$, and heart rate was $77.63 \pm 9.78$ beats per minute and systolic and diastolic blood pressure were $110.99 \pm 12.84$ and $71.80 \pm 8.37 \mathrm{mmHg}$, respectively. Before the VVS diagnosis, the number of syncope episodes was eight (6-14). The timing of the syncope episode during the tilt table test was $29.69 \pm 13.07$ minutes, and the duration of asystole was $11(9-19)$ seconds. The number of patients in type-1, type-2 and type-3 VVS was 43 (49), 15 (17), 30 (34), respectively. Scales were determined as an EQ-5D index 0.23 (0.10-0.61), EQ-5D-VAS index 73.64 \pm 14.39 , Beck-depression 19.42 \pm 8.75 and Beckanxiety16.36 \pm 7.00 (Table 1 ).
Table 2 shows the demographic and clinical characteristics of the patients with vasovagal syncope types. There was no statistically significant difference between the VVS types and EQ-5D index, EQ-5D-VAS index, Beck-depression, and Beck-anxiety scales $(p=0.47$, $\mathrm{p}=0.48, \mathrm{p}=0.75, \mathrm{p}=0.15)$. In correlation analyze, there was no significant correlation between the timing of the syncope episode, duration of asystole and EQ-5D index, EQ-5D-VAS index, Beck-depression, and Beck-anxiety scales. The relationship between the total number of syncope episodes (TSE) and EQ-5D index, EQ-5D-VAS index, Beck-depression, and Beck-anxiety scales was analyzed using Pearson correlation. There was a significant and negative correlation between the TSE and EQ-5D index and EQ-5D-VAS index, respectively $(p<0.001$, $\mathrm{r}:-0.649 ; \mathrm{p}<0.001, \mathrm{r}:-0.587)($ Fig. 1A, B). There was a significant and positive correlation between TSE and Beck-anxiety scale ( $p<0.001, r \div 0.622)$ (Fig. 1C). Moreover, TSE was significantly and positively correlated with the Beck-depression scale ( $p<0.001, r \div 0.755)$ (Fig. 1D).

The relationship between TSE and independent parameters (age, BMI, gender, ejection fraction, heart rate, SBP, DBP, EQ-5D index, EQ-5D-VAS index, Beck-depression and Beck-anxiety scales) was analyzed with linear regression models. EQ-5D index, EQ-5D-VAS index, Beck-depression scale and Beck-anxiety scales were defined as an independent parameter of TSE in regression analyze (Table 3 ). 
TABLE 3. Factors related to TSE of VVS patients in stepwise multiple linear regression analysis

Coefficients

\begin{tabular}{|c|c|c|c|c|}
\hline \multirow[t]{2}{*}{ Model } & \multicolumn{2}{|c|}{ Unstandardized coefficients } & \multirow{2}{*}{$\frac{\text { Standardized coefficients }}{\text { Beta }}$} & \multirow[t]{2}{*}{$\mathrm{p}$} \\
\hline & B & Std. error & & \\
\hline (Constant) & 8.237 & 2.272 & & $<0.001$ \\
\hline EQ-5D index & -3.009 & 1.270 & -0.178 & 0.020 \\
\hline EQ-5D vas & -0.052 & 0.024 & -0.154 & 0.030 \\
\hline Beck-depression & 0.124 & 0.055 & 0.221 & 0.026 \\
\hline Beck-anxiety & 0.264 & 0.064 & 0.441 & $<0.001$ \\
\hline
\end{tabular}

SD: Standard deviation.

\begin{tabular}{lcccr}
\hline & \multicolumn{4}{c}{ Excluded variables } \\
\cline { 2 - 4 } Model & $\mathrm{B}$ & Partial correlation & Collinearity statistics & $\mathrm{p}$ \\
\cline { 3 - 4 } & & & Tolerance & 0.556 \\
Age & 0.040 & 0.065 & 0.971 & 0.342 \\
Women & 0.068 & 0.104 & 0.841 & 0.223 \\
BMI & 0.081 & 0.134 & 0.973 & 0.855 \\
SBP & 0.012 & 0.020 & 0.973 & 0.794 \\
DBP & 0.018 & 0.029 & 0.978 & 0.633 \\
Heart rate & 0.032 & 0.052 & 0.752 & 0.412 \\
Ejection fraction & 0.075 & 0.121 & & \\
\hline
\end{tabular}

a. Dependent variable: TSE; b. Correlates in the model: (Constant), EQ-5D index, EQ-5D VAS, Beck-depression, Beck- anxiety. TSE: Total syncope episodes; BMI: Body mass index; SBP: Systolic blood pressure; DBP: Diastolic blood pressure.

\section{DISCUSSION}

In this study, we studied the relationship between TSE and psychological status and quality of life in patients with VVS. This study demonstrated three major findings, as follows:

1 - There was a significant and negative correlation between the TSE and EQ-5D and EQ-5D-VAS indexes.

2- TSE was correlated significantly and positively with Beck-depression and Beck-anxiety scales.

3- EQ-5D index, EQ-5D-VAS index, Beck-depression and Beck-anxiety scales were independent parameters of TSE.

VVS, which is a recurrent and potentially debilitating medical disorder, is characterized by a high tendency to fainting, and it affects the patients' quality of life [23]. Although the pathophysiology of VVS has not been un- derstood clearly, peripheral autonomic dysfunction and central serotonergic mechanisms are the essential causes of vasovagal reflex $[24,25]$. Also, the serotonin molecule plays a vital role in the pathophysiology of VVS, and it was suggested that similar pathophysiological features could be between depression and VVS [25]. Fear may increase the risk of physiological syncope symptoms due to similar autonomic symptoms of syncope and anxiety [26]. The existence of anxiety and depression in more than $30 \%$ of the VVS patients confirms this suggestion [16]. Also, VVS episodes are affected by psychosocial factors, such as anxiety, depression, personal beliefs about psychological disease, and fear $[16,27]$.

Previous studies showed that there is a relationship between psychological disorders and cardiovascular disease. Koponen et al's studydemonstrated that depressive symptoms associated with increased CV mortality and morbidity risk, especially in men [28]. Also, the prognostic significance of depression has been demonstrated 

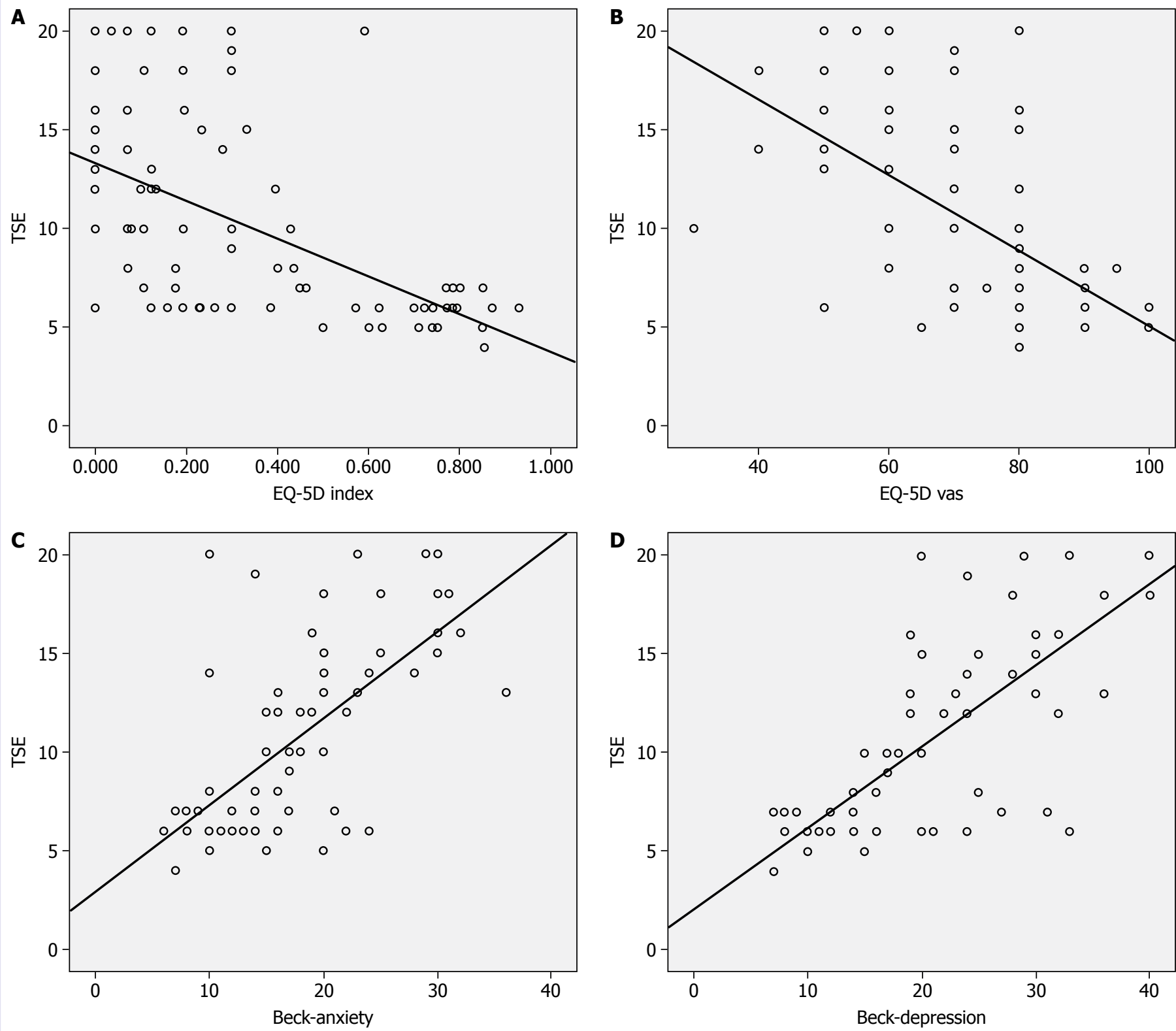

FIGURE 1. (A) The correlations between the total syncope episodes (TSE) and the EQ-5D index in VVS (Vasovagal Syncope) patients. (B) The correlations between the total syncope episodes (TSE) and EQ-5D-VAS index in VVS (Vasovagal Syncope) patients.

(C) The correlations between the total syncope episodes (TSE) and the Beck-anxiety scale in VVS (Vasovagal Syncope) patients.

(D) The correlations between the total syncope episodes (TSE) and Beck-depression scale in VVS (Vasovagal Syncope) patients.

in patients with atrial fibrillation, heart failure, and coronary artery disease [29]. Moreover, previous studies have reported a relationship between psychological stress and recurrent syncope episodes in VVS patients $[15,16,27,30]$. Anderson et al. found that the recurrent syncope episodes impaired the quality of life and initiated depressive symptoms [31]. It has also been reported that the psychiatric disorder prevalence was $80 \%$ in patients with syncope $[15,30,32]$. Recently, a prospective study found that $45 \%$ of adult patients with depression or anxiety disorders had a history of syncope episode [33]. Similarly, anxiety [34], depression [35] and somatization disorder [30] prevalence were reported in patients with positive tilt table test and recurrent syncope episodes. However, the association between the syncope severity and impaired quality of life is still unclear, and our findings may find an answer to this uncertainty. In our study, we aimed to find an association between the 
psychological status and VVS pathophysiology, and we showed that EQ-5D index, EQ-5D-VAS index, Beckdepression and Beck-anxiety scales were an independent predictor of TSE, and there was a significant correlation between the TSE and these scales.

The lack of a control group in our study makes it open to discussion. However, the EQ-5D index, EQ-5D-VAS index, Beck-depression and Beck-anxiety scales were previously studied in the Turkish population, so these findings eliminate the discussion [36-38]. The EQ-5D index, EQ-5D-VAS index, Beck-depression and Beckanxiety scales' scores were reported from previous studies in the Turkish population from various diseases. These scores were defined as a $0.91,87,8,6$ for the EQ-5D, EQ-5D-VAS, Beck-depression and Beck-anxiety scales, respectively [39-41]. The different scale scores between the present study and previous published papers in the Turkish population confirm our hypothesis. On the other hand, it is not well-known whether the recurrent syncope episodes were a reason or a consequence for psychiatric disorders. The number of syncope episodes increased in patients with psychiatric disorders, and recurrent syncope episodes might harm the psychosocial status $[15,30,42]$. The frequency of syncope episodes was less frequent or recovered entirely in patients under psychiatric treatment than those who did not receive such treatment $[32,43]$. On the other hand, it was reported that VVS patients with unresponsive to conventional treatment had higher psychological discomfort [27]. Higher psychosocial discomfort levels could predict resistance to conventional treatment in VVS patients [44]. Also, SSRIs had a positive effect on the refractory recurrent syncope episodes $[12,45]$. A recent study demonstrated that fluoxetine could be considered as the first-line treatment in VVS patients with recurrent syncope episodes and psychogenic disorders [46]. Similarly, Kadri et al. observed a significant improvement with citalopram treatment in syncope patients when the primary endpoint was considered as a well-being score (WS) [47]. Because syncope might occur secondary to psychiatric disorders, these findings showed the importance of evaluation of the psychiatric status. To support this theory, the American Psychiatric Association included syncope as one of the symptoms of panic, somatization, and phobic disorders [48]. As in our study, psychiatric disorders could predict the frequency of syncope episodes $[15,30,42]$, and detecting psychiatric problems with scales might help to determine the psychological status and syncope treatment options.
When contemplating the high prevalence of psychological disorders and the role of beliefs about VVS, psychotherapies might be useful in VVS patients. Previous case reports showed that physical activities, such as muscle strengthening, and cognitive-behavioral therapies, were effective in the treatment of the VVS patients [49-51]. Moreover, serotonin molecule plays a vital role in VVS pathophysiology, and SSRIs are useful in the treatment of VVS episodes. It is hypothesized that SSRIs affect by inhibiting sympathetic activity in central neurons [52].

\section{Limitations}

This present study has some limitations. This was a single-center study and based on a relatively small group of patients. One other limitation of this study was the lack of physiatrists. The data were collected from subjective and self-reported scales, and it may be possible bias or misinterpretation. Thus, we used the standard forms, which are accepted by the previous literature, to minimize the misinterpretation and increase the reliability of the data. Also, our study has shown the relationship between VVS and psychological disorders, but we could not distinguish whether the psychological disorder was a reason or consequence. These findings could not be generalized to all patients with syncope due to VVS patients were examined, and data were collected just in the arrhythmia clinics in this study. The lack of healthy subjects was another limitation of this study.

\section{Conclusion}

In conclusion, this study showed that psychological factors play an essential role in patients with VVS. Many psychological problems seem to be crucial in the development and prevention of VVS-related injuries. It has been shown that conventional treatment was ineffective in VVS patients with psychological problems [27]. Early diagnosis and treatment are crucial to managing syncope episodes in patients with psychological problems. Clinicians should be aware of the effects of psychological factors in syncope and should find new treatment strategies. Therefore, psychological evaluation of syncope patients might be helpful in preventing future syncope attacks. Pharmacological treatment could be useful, as well as psychological therapy, for anxiety or depression in VVS patients, and it may decrease the frequency of syncope episodes. Further studies are needed to identify effective treatments in VVS patients. 
Ethics Committee Approval: Istanbul University, Istanbul Faculty of Medicine Clinical Research Ethics Committee approved the study, and all patients gave informed consent (date: 18/01/2016; number: 20302).

Informed Consent: Informed consent was obtained from all individual participants included in the study.

Conflict of Interest: No conflict of interest was declared by the authors.

Financial Disclosure: The authors declared that this study has received no financial support.

Authorship Contributions: Concept - AA, AD; Design - RA, AKB; Supervision - IY, NGS; Fundings - BE, RS; Materials - AD, BE; Data collection and/or processing - AT, RA; Analysis and/or interpretation - AT, AKB; Literature review - IY, BE; Writing - AT, AD; Critical review - RA, AKB.

\section{REFERENCES}

1. Vaddadi G, Lambert E, Corcoran SJ, Esler MD. Postural syncope: mechanisms and management. Med J Aust 2007;187:299-304. [CrossRef]

2. Savage DD, Corwin L, McGee DL, Kannel WB, Wolf PA. Epidemiologic features of isolated syncope: the Framingham Study. Stroke 1985;16:626-9. [CrossRef]

3. Ganzeboom KS, Mairuhu G, Reitsma JB, Linzer M, Wieling W, van Dijk N. Lifetime cumulative incidence of syncope in the general population: a study of 549 Dutch subjects aged 35-60 years. J Cardiovasc Electrophysiol 2006;17:1172-6. [CrossRef]

4. Mark AL. The Bezold-Jarisch reflex revisited: Clinical implications of inhibitory reflexes originating in the heart. J Am Coll Cardiol 1983;1:90-102. [CrossRef]

5. Almquist A, Goldenberg IF, Milstein S, Chen MY, Chen XC, Hansen $\mathrm{R}$, et al. Provocation of bradycardia and hypotension by isoproterenol and upright posture in patients with unexplained syncope. N Engl J Med 1989;320:346-51. [CrossRef]

6. Perna GP, Ficola U, Salvatori MP, Stanislao M, Vigna C, Villella A, et al. Increase of plasma beta endorphins in vasodepressor syncope. Am J Cardiol 1990;65:929-30. [CrossRef]

7. Quan KJ, Carlson MD, Thames MD. Mechanisms of heart rate and arterial blood pressure control: implications for the pathophysiology of neurocardiogenic syncope. Pacing Clin Electrophysiol 1997;20:764-74.

8. Bayram E, Kir M, Topçu Y, Akinci G, Hiz S, Sağın Saylam G. Retrospective Evaluation of the Patients Who Admitted with Syncope. Turkiye Klinikleri J Pediatr 2011;20:210-3.

9. Lofving B. Cardiovascular adjustments induced from the rostral cingulate gyrus with special reference to sympatho-inhibitory mechanisms. Acta Physiol Scand Suppl 1961;53:1-82.

10. Rea RF, Thames MD. Neural control mechanisms and vasovagal syncope. J Cardiovasc Electrophysiol 1993;4:587-95. [CrossRef]

11. Thompson WO, Thompson PK, Dailey ME. The effect of posture upon the composition and volume of the blood in man. J Clin Invest 1928;5:573-604. [CrossRef]

12. Di Girolamo E, Di Iorio C, Sabatini P, Leonzio L, Barbone C, Barsotti A. Effects of paroxetine hydrochloride, a selective serotonin reuptake inhibitor, on refractory vasovagal syncope: a randomized, double-blind, placebo-controlled study. J Am Coll Cardiol 1999;33:1227-30. [CrossRef]

13. Kapoor WN, Fortunato M, Hanusa BH, Schulberg HC. Psychiatric illnesses in patients with syncope. Am J Med 1995;99:505-12. [CrossRef]
14. Linzer M, Pontinen M, Gold DT, Divine GW, Felder A, Brooks WB. Impairment of physical and psychosocial function in recurrent syncope. J Clin Epidemiol 1991;44:1037-43. [CrossRef]

15. Kouakam C, Lacroix D, Klug D, Baux P, Marquié C, Kacet S. Prevalence and prognostic significance of psychiatric disorders in patients evaluated for recurrent unexplained syncope. Am J Cardiol. 2002;89(5):530-535. [CrossRef]

16. Shaffer C, Jackson L, Jarecki S. Characteristics, perceived stressors, and coping strategies of patients who experience neurally mediated syncope. Heart Lung 2001;30:244-9. [CrossRef]

17. Linzer M, Varia I, Pontinen M, Divine GW, Grubb BP, Estes NA 3rd. Medically unexplained syncope: relationship to psychiatric illness. Am J Med 1992;92:18S-25S. [CrossRef]

18. Writing Committee Members, Shen WK, Sheldon RS, Benditt DG, Cohen MI, Forman DE, et al. 2017 ACC/AHA/HRS guideline for the evaluation and management of patients with syncope: A report of the American College of Cardiology/American Heart Association Task Force on Clinical Practice Guidelines and the Heart Rhythm Society. Heart Rhythm 2017;14:e155-e217.

19. Passman R, Horvath G, Thomas J, Kruse J, Shah A, Goldberger J, et al. Clinical spectrum and prevalence of neurologic events provoked by tilt table testing. Arch Intern Med 2003;163:1945-8. [CrossRef]

20. Sutton R, Petersen M, Brignole M, Raviele A, Menozzi C, Giani P. Proposed classification for tilt induced vasovagal syncope. Eur J Cardiac Pacing Electrophysiol 1992;3:180-3.

21. Drummond MF, Sculpher MJ, Claxton K, Stoddart GL, Torrance GW. Methods for the economic evaluation of health care programmes.4th ed. Oxford University Press; 2015.

22. Beck AT, Steer RA, Carbin MG. Psychometric properties of the Beck Depression Inventory: Twenty-five years of evaluation. Clin Psychol Rev 1988;8:77-100. [CrossRef]

23. Grubb BP, Kosinski D. Current trends in etiology, diagnosis, and management of neurocardiogenic syncope. Curr Opin Cardiol 1996;11:3241. [CrossRef]

24. Theodorakis GN, Livanis EG, Leftheriotis D, Flevari P, Markianos M, Kremastinos DT. Head-up tilt test with clomipramine challenge in vasovagal syndrome--a new tilt testing protocol. Eur Heart J 2003;24:658-63. [CrossRef]

25. Hainsworth R. Pathophysiology of syncope. Clin Auton Res. 2004;14 Suppl 1:18-24. [CrossRef]

26. Rapee RM, Sanderson WC, McCauley PA, Di Nardo PA. Differences in reported symptom profile between panic disorder and other DSMIII-R anxiety disorders. Behav Res Ther 1992;30:45-52. [CrossRef]

27. Gracie J, Newton JL, Norton M, Baker C, Freeston M. The role of psychological factors in response to treatment in neurocardiogenic (vasovagal) syncope. Europace 2006;8:636-43. [CrossRef]

28. Koponen H, Jokelainen J, Keinänen-Kiukaanniemi S, Vanhala M. Depressive symptoms and 10-year risk for cardiovascular morbidity and mortality. World J Biol Psychiatry 2010;11:834-9. [CrossRef]

29. Hare DL, Toukhsati SR, Johansson P, Jaarsma T. Depression and cardiovascular disease: a clinical review. Eur Heart J 2014;35:1365-72.

30. Giada F, Silvestri I, Rossillo A, Nicotera PG, Manzillo GF, Raviele A. Psychiatric profile, quality of life and risk of syncopal recurrence in patients with tilt-induced vasovagal syncope. Europace 2005;7:465-71.

31. Anderson JB, Czosek RJ, Knilans TK, Marino BS. The effect of paediatric syncope on health-related quality of life. Cardiol Young 2012;22:583-8. [CrossRef]

32. Ventura R, Maas R, Rüppel R, Stuhr U, Schuchert A, Meinertz T, Nienaber CA. Psychiatric conditions in patients with recurrent unex- 
plained syncope. Europace 2001;3:311-6. [CrossRef]

33. Leftheriotis D, Michopoulos I, Flevari P, Douzenis A, Koborozos C, Kostopoulou A, et al. Minor psychiatric disorders and syncope: the role of psychopathology in the expression of vasovagal reflex. Psychother Psychosom 2008;77:372-6. [CrossRef]

34. Cohen TJ, Thayapran N, Ibrahim B, Quan C, Quan W, von zur Muhlen F. An association between anxiety and neurocardiogenic syncope during head-up tilt table testing. Pacing Clin Electrophysiol 2000;23:837-41.

35. McGrady A, Kern-Buell C, Bush E, Khuder S, Grubb BP. Psychological and physiological factors associated with tilt table testing for neurally mediated syncopal syndromes. Pacing Clin Electrophysiol 2001;24:296-301. [CrossRef]

36. Hisli N. Beck Depresyon Envanteri’nin gecerliliği üzerine bir calışma. Psikoloji Dergisi 1988;6:18-122.

37. Ulusoy M, Sahin NH, Erkmen H. Turkish version of the Beck Anxiety Inventory: psychometric properties. Journal of Cognitive Psychotherapy $1998 ; 12: 163-72$.

38. Hoopman R, Muller MJ, Terwee CB, Aaronson NK. Translation and validation of the EORTC QLQ-C30 for use among Turkish and Moroccan ethnic minority cancer patients in the Netherlands. Eur J Cancer 2006;42:1839-47. [CrossRef]

39. Bilgin MS, Özenç S, Sarı O, Yeşilkaya Ş, Aydoğan Ü, Koç B. A Study on Quality of Life in Patients with Diabetes J Clin Anal Med 2015;6:15963.

40. Atasoy V, Anaforoğlu İ, Algün E, Kutanis R. Depression, Anxiety and Quality of Life Among Adult Turkish Patients with Type 1 Diabetes Mellitus. Turk Jem 2013;17:28-32. [CrossRef]

41. Karkucak M, Özden G, Çapkin E, Tosun M, Alaca H, Barçak Ö. Factors Affecting the Quality of Life, Anxiety and Depression Levels in Ankylosing Spondylitis. F.Ü. Sağ Bil Tip Derg 2010:24:13-9.

42. D’Antono B, Dupuis G, St-Jean K, Lévesque K, Nadeau R, Guerra P, et al. Prospective evaluation of psychological distress and psychiatric morbidity in recurrent vasovagal and unexplained syncope. J Psychosom Res 2009;67:213-22. [CrossRef]

43. Newton JL, Kenny RA, Baker CR. Cognitive behavioural therapy as a potential treatment for vasovagal/neurocardiogenic syncope--a pilot study. Europace 2003;5:299-301. [CrossRef]

44. Flint B, Baker C, Freeston M, Newton JL. Level of psychosocial impairment predicts early response to treatment in vasovagal syncope. Europace 2009;11:231-6. [CrossRef]

45. Vyas A, Swaminathan PD, Zimmerman MB, Olshansky B. Are treatments for vasovagal syncope effective? A meta-analysis. Int J Cardiol 2013;167:1906-11. [CrossRef]

46. Flevari P, Leftheriotis D, Repasos E, Katsaras D, Katsimardos A, Lekakis J. Fluoxetine vs. placebo for the treatment of recurrent vasovagal syncope with anxiety sensitivity. Europace 2017;19:127-31. [CrossRef]

47. Kadri AN, Nusairat L, Kadri S, Alqaid A, Hernandez AV, Kadri NN. Effect of Citalopram for the Treatment of Neurocardiogenic Syncope. Am J Ther 2019;26:e339-43. [CrossRef]

48. American Psychiatric Association. Diagnostic and Statistical Manual of Mental Disorders (DSM-5). American Psychiatric Pub; 2013. [CrossRef]

49. Sabin N. The use of applied tension and cognitive therapy to manage syncope (common faint) in an older adult. Aging Ment Health 2001;5:92-4. [CrossRef]

50. Van Dijk N, Velzeboer SC, Destrée-Vonk A, Linzer M, Wieling W. Psychological treatment of malignant vasovagal syncope due to bloodphobia. Pacing Clin Electrophysiol 2001;24:122-4. [CrossRef]

51. Anderson KW, Taylor S, McLean PD. Panic disorder associated with blood-injury reactivity: The necessity of establishing functional relationships among maladaptive behaviors. Behav Ther 1996;27:463-72.

52. Grubb B. Serotonin and syncope: an emerging connection? Eur J Card Pacing Electrophysiol 1996;5:306-14. 